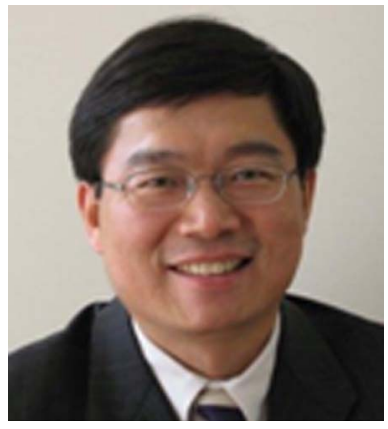

\section{JBO Is Going Monthly}

A phone book in the mailbox? Recent issues of our Journal of Biomedical Optics have been as thick as an inch $(2.5 \mathrm{~cm})$. Having experienced continuous rapid growth, JBO has been publishing $>300$ papers each year since 2008 (Fig. 1). While celebrating the expansion, we recognize that the bimonthly printed issues are unwieldy as well as untimely. Although papers are posted online as soon as they are typeset and approved, we would like to get the printed issues into the hands of our subscribers more frequently and in a more manageable size. Believe it or not, some of us still like to read hard copies or carry them on flights. One of the ten initiatives I made earlier this year was to publish JBO monthly, and this has now been officially approved by SPIE. Starting January 2011, we will see more timely and frequent monthly issues.

The growth of the journal is in line with the growth of the field of biomedical optics (Fig. 2). Photonics West BiOS, the largest conference on biomedical optics, grew from 606 papers presented in 1996 to 1776 papers expected for 2011. Our field has ushered in a host of exciting technological innovations and biomedical applications-ranging from optical imaging of gene expression, to optical coherence tomography of the retina, to laser therapy of port wine stains. As the only part of the electromagnetic spectrum that reflects molecular structures, light waves are expected to find more and more unique applications in biomedicine.

We strive to provide a competitive publication platform for biomedical optics. Our review cycles, especially for Letters, have been shortened significantly without sacrificing the quality of reviews. Notably, all Letters enjoy immediate open access without mandatory publication fees. Authors are en-

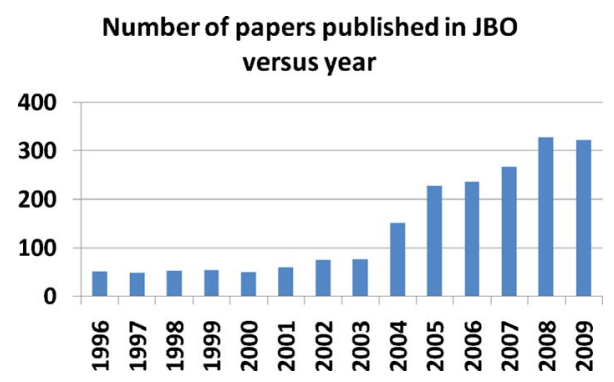

Fig. 1 Number of papers published in JBO versus year. couraged to take advantage of this generous policy made possible by SPIE.

On another topic, I would like to let our readers know about a matter related to the impact factor of JBO. SPIE has provided the following statement regarding the 2009 impact factors:

When the 2009 impact factors (IFs) were released in June 2010, SPIE observed that the aggregate IF for the Optics subject category dropped by $8.8 \%$ from 2008 to 2009 and that the IFs for SPIE journals had decreased even more than that. In a review of these results, SPIE has identified a significant contributor to these declines.

Historically SPIE Proceedings were indexed in the ISI Index to Scientific and Technical Proceedings (now part of Thomson Reuters) and are now included in the Web of Science ${ }^{\mathrm{TM}}$ Conference Proceedings Citation Index. However, due to an administrative misunderstanding, the SPIE Proceedings published in 2009 were not indexed before the 2009 Journal Citation Report (JCR) metrics were calculated. This delay in coverage of SPIE Proceedings in Web of Science ${ }^{\mathrm{TM}}$ appears to be a major reason for the lower Optics impact factors.

SPIE Proceedings account for a significant number of cites to JCR Optics category journals. The delay of coverage of 2009 SPIE Proceedings in Web of Science ${ }^{\mathrm{TM}}$ means that these citations were not in-

\section{Number of papers presented in BiOS versus year}

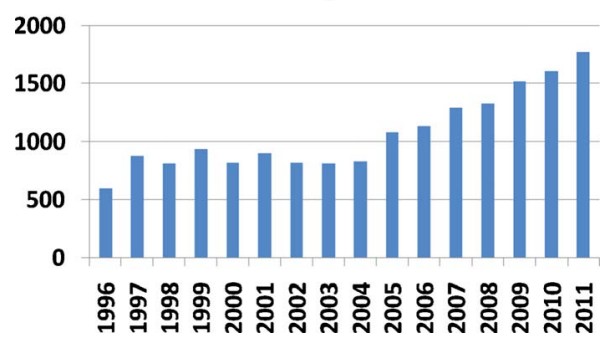

Fig. 2 Number of papers presented in Photonics West BiOS versus year. 
cluded in the 2009 IF calculations. Because SPIE journals are cited relatively frequently in SPIE Proceedings, their 2009 IFs likely were impacted to a greater degree than some other Optics journals.

SPIE and Thomson Reuters have taken steps to correct the situation. The 2010 impact factors will include citations from the SPIE Proceedings published in 2010. Thomson Reuters is working to complete indexing the SPIE Proceedings papers published during the gap in coverage so that all SPIE Proceedings will be covered in Web of Science ${ }^{\mathrm{TM}}$ and be available to researchers as well as included in other article-specific citation metrics, such as h-index. However, the 2009 JCR metrics, including impact factor, cannot be recalculated.

Questions about this matter may be directed to Eric Pepper, SPIE Director of Publications, at eric@spie.org.
Lihong V. Wang, Ph.D. Editor-in-Chief 